Some ten days later I examined him again, and on this occasion a presystolic murmur was audible, brief and soft but decisive. Oddly enough, now the thrill had vanished; again and again $I$ tried to find it but in rain. So capricious is this clisease in its phyjsical signs, and so elusive are its signs, in respect of military service or of life insurance. I am not as yet ready to accept a definite distribution of stenosis murmurs into early, middle, and late. Again, a young friend of mine, very active both in mind and body, and anxious to serve in the army, has been rejected on two occasions for "soldier's heart." He has a slight addi. tion to the short first sound and a short presystolic thrill. He has never had rheumatic fever nor other illness.

We have had a complicated illustration of this pliase in the Mount Vernon Hospital. With Dr. Wilson and another colleague I examined a man with the obvious signs of aortic regurgitation. The question arose whether or no a divided first sound, audible at the apex only, signified a reduplication of the first sound. This it scarcely seemed to be; the taps were too close and the first one too light and brief; but I thought I recognized in it such a split in the first sound as I have described. The carotid beat being, as usual in such cases, only too definite, we were able to conclude that the first tap of the duplex sound was presystolic. Following this up wo detected a pre: systolic thrill, short and light but quite distinct.

A thrill must be very carefully placed and timed, for in many of these irritable hearts a systolic thrill can be felt; perhaps in most of them if some effort be made just before examination. It is short but often very distinct to the touch. It is not felt about the base of the heart, and is therefore not due to a slack aorta. How the thrill is generated it is not easy to say. Very often in these cases a systolic murmur at the apex is recorded, an alteration of the first sound, more like a prolongation of it with some change of quality than like a definite murmur of mitral regurgitation. 'I'o suggest the cause of this thrill is but to guess; but, if I may guess, my suggestion would be that in these cases the papillary muscles and their chords may be a trifle slack, so that the mitral sheets shiver a little in the current, as a sail shivers when the boat gets near the wind. Such a quaver would be transmitted to the ventricular wall, and thence to the thoracic. In such a condition there might well be a minute, inconstant, and insignificant regurgitation also; though ordinarily in mitral regurgitation there is no thrill.

In conclusion, I lope it may not be unbecoming in me as a member of the staff, but rather an onlooker than of effectual service, to offer my tribute of praise to the author of this Report; a brief summary of infinite pains, of his own and of those of his colleagues who are and have been on resident duty at the hospital. REFERENCEs.
"Medical Research Committee's Report upon Soldiers returned as
Cases of "Disordered Action of the Heart," or "Valvular Disease of
the Heart." Spec. Rept. Ser. No 8. 2 Zeit. f. klin. Med. 8 Seo
C. Allbutt's Diseases of the Arteries, ii, 475 .

\section{ON GUNSHOT INJURIES OF THE CHEST,}

With Especial Reference to Haemothorax.

BY

Sir JOHN ROSE BRADFORD, K.C.M.G., C.B., F.R.S., Temporary Colonel A.M.S., Consulting Physician. BRITISH EXPEDITIONARY ForCE.

Wounds of the chest do not as a rule reach the bases in France until the fourth day after the infliction of the wound, except in periods of heavy fighting, then they may arrive much earlier, that is, on the second day. 'The observations recorded in this paper deal more especially with cases observed from the third or fourth day up to about three weeks after the occurrence of the injury, and thus they deal with only a portion of the subject. Gunshot wounds of the chest are usually divided into those penetrating and involving the lungs or other thoracic viscera, and the non-penetrating, although it is not always possible to be certain during life that an apparent non-penetrating wound is really such. Non-penetrating wounds may, however, give rise to pleural and pulmonary lesions, and in a certain proportion of cases of simple clean perforating ballet wounds of the chest the positions of the entry and exit wounds suggest that the bullet does not only pass through the chest but also through the lung, nevertheless there are no signs or symptoms indicating the presence of either air or fluid in the pleura, and such cases may recover rapidly and apparently completely. Pleurisy and empyema are the most common pleural lesions produced by wounds of the chest wall not involving the lung. Tho pleurisy is often at first dry, but not uncommonly an effusion follows, and since such lesions are often due to streptococcal infoctions spreading from the wound track in the chest wall to the pleura, an empyema of the usual type derelops and requires the usual treatment.

Haemoptysis, haemorrhagic infiltration of the lungs, and pneumonia of a septic bronchopneumonic type are the pulmonary lesions that may follow on a wound limited to the chest wall. In addition to these, collapse of the lung of a massive type may occur on the side opposite to the injury even when the latter causes no pleural or pulmonary lesion on the side injured. The haemoptysis and the septic pneumonia are both directly associated with the bruising and consequent bleeding into the lung pro. duced by the impact of the missile on the chest wall and the resulting injury to the cliest wall and lung. Thie haemoptysis may not only be severe, but may also run a long course, and some of the most serere cases of haemoptysis seen by the writer in a very large series of chest cases were those in which the missile only bruised the chest and had not only not produced any wound; but where there was no evidence of any fractured ribs.

The bruised and blood infiltrated lung is very prone to become infected either from the lung or by the spread of infection from the wound track in the chest wall, and hence pneumonia of septic or of a bronchopneumonic type develops. In some cases wounds of the chest wall lay open the pleural cavity but do not involve the lung, a portion of the chest wall, more especially in its lower parts or at the back, is carried away and a gaping hole, often of considerable size, is left. In such cases the lung does not as a rule unjergo complete collapse, although the pleural cavity is in free communication with the air. In fact, the volume of the lung seems to approximate to that seen after death when the pleura is open, and therefore thie pulmonary collapse is markedly less than that seen in ordinary pneumothorax where, as is well known, very complete collapse occurs, yet in the latter cases the intrapleural pressure may not be above that of the atmosphere.

Penetrating wounds of the chest usually produce one or more of the following conditions : subeutaneous emphysema, haemothorax, pneumothorax, laceration of the lung. These conditions may not only arise where the wound is one obviously involving the chest, or where the wound of entry is near the cliest-for example, the neck or the abdomen especially in its upper part; but wounds in other regions-for example, the face or arm-may also give rise to haemothorax or pneumothorax from the course of the bullet being peculiar owing to the position of the soldier at the moment he is struck. In such cases there is often only a wound of entry, and this may be small and inconspicuous, but yet a large haemothorax or eren a pneumothorax may be present.

Further, in wounds of the chest the haemothorax or pneumothorax may be on the side opposite to the wound of entry; the missile taking an oblique course may enter on one side, more especially in wounds of the back, and follow such a course that it does not open the pleural cavity on the side of entry, although opening that on the opposite side without or with a wound of exit. Again, in some instances a bullet may enter and leave the pleural cavity on one side without causing any appreciable lesion on that side, and yet so wound the pleura and lung on the opposite side as to cause marked lesions.

Bilateral haemothorax is sometimes seen as a result of a single wound the track of which is transverse or oblique, and haemopericardium may occur in association with either unilateral or bilateral haemothorax.

Unilateral wounds, however, may cause bilateral effects owing to the development of contralateral collapse or contralateral pneumonia.

The subcutaneous or so.called surgical emphysema so often present in chest wounds is usually limited to the vicinity of the wound or to an area of the chest wall adjacent of variable size. In exceptional instances it; 
distribution is more extensive, and it may sometimes involve not only the trunk but even the whole body, including the face and extremities.

\section{HaEnothoRax.}

Haemothorax is the most common result of a chest wound; both pneumo-haemothorax and pneumothorax are relatively rare, and in a series of 328 cases of gunshot wounds of the chest only 8 cases of the former and 4 cases of the latter were observed.

The haemothorax may be either sterile or infected, and if the latter the infection may be either early or late. If early, the infection oecurs within the first fow days after the infliction of the wound, and is doubtless dependent upon infective material being not only carried in with the missile, but directly infecting the effused blood generally and rapidly. In such cases exploration of the haenothorax reveals the presence of organisms in the fluid withdrawn Infection, however, may occur later, that is, in the second or third week after the receipt of the wound. Such cases may at first run the course of a sterile haemothorax, with but fow symptoms, and the fluid removed by paracentesis may fail to show the presence of any organisms. Suddenly urgent symptoms, such as increasing fever, dyspnoea, pain, rapid pulse and jaundice, arise, and paracentesis then reveals the presence of organisms in the fluid withdrawn. The infection may be more or less localized, and more especially so in the lower portions of the chest, and in such cases paracentesis may yield different results according to the site explored; at one spot a sterile fluid may be obtained and at another at no great distance from the first a fluid of a totally different character containing abundant organisms. This is especially the case with anaërobic bacillary infections. It is probable that in many cases the explanation of the rapid development of the clinical picture of infection in the course of what seemed for some days to be a case of simple sterile haemothorax, is to be sought in the fact that the infecting agent, that is, fragment of clothing, etc., was shut off by blood clot from the general mass of haemo. thorax fluid in the chest. Later, owing to the spread of infection through the clot, the haemothorax becomes infected as a vliole, and hence the rapid development of urgent and very dangerous symptoms. In a series of 450 cases of haemothorax observed by Bradford and Elliott infectiou was present in 117-that is to say, roughly, in 25 per cent.; and Captain H. Henry, who carried out the bacteriological investigation, found that lung organisms such as the pneumococcus; $B$. influenzae and $M$. tetragenus, were present in some 20 per cent. of the infected cases, and that in the remaining 80 per cent. streptococci, staphylococci, and anaërobic gas forming bacilli were found.

In approximately 50 per cent. of the infected cases anaërobic bacilli, either alone or in association with cocci, were present; lience it would seem that infection of a haemothorax is much more frequently derived from the skin or clothing than directly from the lung. In some instances the pleural infection arises from direct extension from the infected wound track in the chest wall, and such a mode of spread may lead to the development of pleural and pulmonary complications in cases of wounds limited to the chest wall, and not directly involving either the pleura or the lung.

Henry and Elliott ${ }^{2}$ have studied the morbid anatomy of wounds of the chest in a series of 100 cases observed in hospital at one of the bases in France. 'These observations deal only with cases that succumbed from the third day to the third week alter the infliction of the wound, and one of the most striking facts ascertained was, that within these limits of time, death from haemorrhage as a result of a wound of the chest was very rare. Thus, in a series of 78 fatal cases of simple haemothorax there were 70 in which either infection or an additional viscéral complication, or bosh, were present. In the remaining eight cases, where death might have been due to haemorrhage, purulent bronchitis was present in three, and in four cases no record existed as to whether sepsis was present or not, thus leaving only one case in which haemorrhage aloue could be definitely asserted to be the sole cause of death.

Septic infection is by far the most frequent, and therefore the most important, cause of death in cases of haemothorax that survive the first few days immediately foll owing the wound.
Secondary haemorrhage is also extremely rare, and in this series of 100 fatal cases only three instances were recorded; in two cases the haemorrlage took place into the pleural cavity, and in one a fatal haemoptysis occurred from a vessel in the vicinity of a small abscess in the lung round a fragment of shell. 'The writer has only seen one other instance of fatal haemoptysis duc to secondary haemorrhage in the course of observations on many hundred cases of gunshot wound of the cliest, and here also it was due to the lesions produced by a fragment of shell embedded in the ling.

The more important points in the morbid anatomy of haemothorax are the following: The degree of collapse of the lung, and especially of the lower lobe, is very marked, and would seem often to be greater in amount than that usually seen in pleural effusions. The over-distension and so-called emphysema of the upper portion of the lung abore the fluid is certainly far greater than that seen in cases of pleural effusion. 'The anterior part of the upper lobe of the lung is frequently pressed up against the chest wall; and the anterior margin of the lung frequently extends beyond the midale liue. There may be a line of loose tibrinous adhesions gluing the surface of the lung to the chest wall at the upper limit of the effused bloood. 'The parietal pleura in the area corresponding to the effused blood is coated with a layer of fibrin one-eighth to one-quarter of an inch thick, and the surface of the collapsed lung is similarly covered to a greater or less extent with a layer of fibrin. The collapsed lung on the side of the haemothorax rarely, if ever, presents signs of inflammation except in the immediate vicinity of the wound track in the lung or round any retained foreign body. Pneumonic consolidation is not found in the lun beneath the haemothorax. Purulent bronchitis may be present not only in the contralateral lung but also in the emphysematous portion of the lung on the side of the haemothorax, but above the fluid. The great collapse of the lung would seem to be not only a safeguard against further haemorrhage in cases where the lung is wounded, but also a very efficient protection against the development of inflammatory complications. There is some evidence to suggest that wounds of the apices of the lungsthat is, the portion that would collapse last-are associated with large collections of bloody fluid in the pleural cavity.

The bloody fluid present in the chest in cases of haemothorax consists in the main of defibrinated blood-that is, a mixture of serum and blood corpuscles, often presenting to the eye a considerable resemblance to blood, but differing from it in that it does not usually undergo massire clotting after removal from the chest. The parietal and visceral pleurae are covered to a greater or less extent with a layer of fibrin, and it is probable that the defibrination of the blood occurs as a result of the movements imparted to it by the respiratory and cardiac movements, and that it usually takes place very soon after the blood is poured out into the chest. Direct experiment has shown that the bloody fluid in haemothorax contains no fibrinogen, and hence, that although it resembles blood to the eye, clotting has really taken place, and that it is in reality defibrinated blood (Elliott and Heñry).

Even in cases of sterile haemothorax the whole of the fluid in the chest is not defibrinated blood. This is shown by two facts: first, the cell content of the fluid is different from that of defibrinated blood in that an increased number of lymphocytes may be present together with endothelial cells, eosinophile cells sometimes in considerable numbers, and marrow cells; secondly, the quantity of fluid is sometimes very large, that is, four to five pints, and even then the patient may not sliow any gross or obvious signs of anaemia. Hence it is evident that there is some pleural exudate as a sequel to, and result of, the presence of the blood in the pleural cavity. In some cases further evidence of the presence of pleural exudate is afforded by the occur. rence of a slight and peculiar clotting in the fluid removed from the chest by paracentesis. This clotting resembles that seen in the fluid of ordinary pleural effusions, but differs from it in that the coagulum contains abundant red corpuscles. This clot, although containing red corpuscles, is often scanty and generally gelatinous in consistence, and hence quite different in appearance from the clot formed from normal entire blood. The superficial resemblance of 
this "secondary clotting," as it is termed by Elliott and Henry, to true clotting is readily explained, inasmuch as the pleural exudate has added coagulable material to the defibrinated blood present in the pleural cavity and the abundant red corpuscles present have been entangled to a greater or less extent in the coagulation. In a small number of cases defibrination of the extravasated blood would seem not to occur, and paracentesis then reveals the presence of a mass of soft jelly-like clot that blocks the needle and prevents satisfactory aspiration; presumably in such cases more or less massive clotting has taken place.

In infected haemothorax the pleural exudate is much more abundant, and hence numerous polymorphonuclear leucocytes are found on microscopic examination, and the fluid removed by exploratory puncture may yield a deposit of pus visible to the naked eye. The fluid itself is often of a crimson colour from the haemolysis that has taken place. Massive clotting would also seem to be more common in infected cases, since, both at operations and in the post-mortem room, large clots may be found, especially in the lower part of the pleural cavity between the diaphragm and the chest wall, and also in the vertebral groove. In these infected cases the inflammatory exudate is apt to be poured out rapidly, and a sudden considerable increase in the amount of fluid in the chest is often strong clinical evidence of the presence of infection, since, as mentioned above, secondary haemorrhage into the pleural cavity in haemothorax is very rare, and then only occurs in infected cases. In sterile cases there is neither secondary haemorrhage nor a sudden copious effusion to cause any sudden great increase in the amount of fluid present in the pleural cavity. In a considerable proportion of these infected cases there is not only a rapid pouring out of inflammatory exudate, but, in addition, there is gas formation, owing to the infection being dependent upon the presence of anaërobic gas-producing organisms. The de velopment of gas is often very rapid, and such cases may present very urgent signs and symptoms that increase rapidly in the course of a few hours. In others the clinical picture is much less urgent, and in a few only* slight symptoms are present, and the condition is only appreciated after careful examination.

The gas may be free in the pleural cavity, associated with very considerable collapse of the lung, and a variable but usually large amount of haemothorax fluid. In other instances the conditions are more peculiar; the collection of gas is localized above the haemothorax fluid, and below the empliysematous over-distended upper lobe of the lung. The loose fibrinous adhesions at the upper level of the fluid in the thorax are probably sufficient to tether the upper lobe to the chest wall, but whether this be the correct explanation of the non-collapse of the entire lung or no, the fact remains that the gas forms a localized collection above the fluid of the haemothorax and below the non-collapsed portion of the lung. Such locelized collections of gas are usually in the lateral or postero-lateral regions of the chest, and more especially in its lower portions. The gas is usually offensive, and has either a definite faecal smell or else that of rotten eggs. It is of ten present under considerable pressure, and in the post-mortem room, if a canuula is inserted, the gas issuing from it readily burns, when lighted, with a bluish flame. In cases of this type there is often considerable displacement of the heart, and this displace ment may develop with great rapidity-that is, 2 in. in twenty-four hours. In cases where the gas is free in the pleural cavity, the clinical picture is that of pneumothorax or pyopneumothorax; and where the condition develops rapidly the symptoms are apt to be urgent and the error may be made of regarding the case: as one of progressive pneumothorax, whereas in reality the phenomena are due to the rapid formation of gas under pressure as the result of infection of the haemothorax fluid.

In infected haemothorax the deposit on the surface of the collapsed lung is much more abundant and thicker than in sterile cases, as a thick layer of lymph more or less organized is often present. This is of considerable importance, inasmuch as this deposit hampers the subsequent roexpansion of the lung after the removal of the fluid by drainage of the pleura. The longer the delay in draining the pleura in such cases the greater is the organization of this deposit, and therefore there is much risk of permanent incomplete expansion of the lung. The deposit on the pleura not only causes the re-expansion of the lung to take place more slowly but also less perfectly, hence it is most important that cases of infected haemo: thorax should be treated and thoroughly drained at the earliest possible moment. Bilateral haemothorax is not very uncommon, and such collections are usually of small or moderate size; they may be either sterile or infected, and cases have occurred of bilateral infected haemothorax that have recovered after drainage of both pleural cavities. In bilateral haemothorax one side may be sterile and the other infected, and recovery has here taken placo after aspiration of the sterile and drainage of the infected haemothorax. Bilateral haemothorax may be associated with haemopericardium, or in infected cases pericarditis may occur as a complication. The pericarditis seen in cases of infectect lraemothorax. is probably usually of streptococcal origin, but in some instances an anaërobic infection of the pericardium may occur and then very striking physical signs may be present owing to the presence of gas and fluid in the pericardial sac. Two such cases have fallen under the observation of the writer and both recovered after opening and draining the pericardium. In one the condition was recognized as the result of the percussion of the pericardial area yielding a tympanitic note. when previously a dull note due to the presence of effusion in the pericardium had been present. Pericarditis probably only occurs in infected cases, although pericardial friction and the signs of fluid in the pericardium may be detected in cases of sterile haemothorax, where, in addition to the pleural lesion, there is also the probability of injury to the -pericardium and where $x$-ray examination may reveal the presence of a bullet in close proximity to the heart. The accurate diagnosis of such cases is often difficult, bat it is probable, inasmuch as they often have no symptoms in. dicative of pericarditis, and they recover, that the pericardial physical signs are due to the presence of a haemo. pericardium rather than to a pericarditis with effusion. In the infected cases the presence of pericarditis is a very serious complication; in some there is merely greasy lymph. in others a moderate effusion, or where an anaërobic infection is present the pericardium may contain gas as well as an effusion. If the effusion is at all large in amount it is usually situated in the posterior part of the pericardium, and is therefore liable to produce some degree of pressure an and collapse of the lower lobe of the left lung. In very large effusions the transverse diameter of the pericardium is greatly increased. When the effusion is posterior in position, the heart may still remain in partial contact with the chest wall anteriorly, and thus, owing to the persistence of friction the presence of even a large effusion may be overlooked.

The complications directly associated with haemothorax are few and are most frequently seen in cases of infected haemothorax. Purulent bronchitis, pneumonia, pleurisy, nassive collapse of the lung, and pericarditis are the most common complications. Abscess and gangrene of the lung may occur, but these are dependent not on the hremothorax but on pulmonary lesions produced by the missile. One case of meningitis due to streptococcal infection in association with an infected haemothorax has fallen under my observation, but no case of cerebral abscess.

The relation of pniulent bronchitis to haemothorax is often very doubtful, as it occurs so frequently apart from wounds, but many patients give a history that is suggestive of the onset of the bronchitis after the wound, aithough even in these it may be dependent on exposure. One of the most striking features of its distribution is its absence in that portion of the lung that is collapsed beneath the haemothorax effusion. Pneumonia, in various forms, may be present, but is not cammon, and here again the collapsed lung on the side of the haemothorax is not involved. It may occur on the side opposite to the haemothorax, but care must be taken in the diagnosis, as the physical signs of massive collapse present great resemblance to those of pneumonia, and, as will be mentioned later, contralateral collapse in cases of unilateral chest. wounds is of quite frequent occurrence. There is, however, clear evidence from autopsy that pneumonia on the side opposite to the wound may occur in haemothorax. Septic broncho. pneumonia is common in wounds of the cliest wall not accompanied by haemothorax, where, as a result of the impact of the missile, the lung is bruised and haemorrhage 
into its substance has occurred. Pleurisy; often dry, is also not uncommon on the side opposite to the haemo: thorax; this occurs most often in infected haemothorax, but some instances have been seen where there was no bacteriological proof that the haemothorax was infected. Pleurisy of a serious type, and often progressing to empyema, occurs as a result of injuries to the chest wall of a tangential character and associated often with fracture of one or more ribs. Septic bronchopneumonia may coexist in such cases. The contralateral pleurisy is especially prevalent in cases of streptococcal infection of haemothorax, and may also progress to effusion and the formation of an empyema. Such cases are necessarily very serious, and if, as is not infrequent, pericarditis is also present they are of the utmost gravity.

Source of the Haemorrhage in Haemothorax.

There is some difference of opinion as to whether the injury to the chest wall, or. that of the lung, is the more common cause of the haemorrhage into the pleural cavity, and it is not possible to dogmatize on this subject. Morbid anatomy does not yield any direct evidence of the bleeding having arisen from the chest wall, but it is obvious that it would be very difficult to exclude the possibility of the wound of an intercostal vessel. On the other hand, it is not unusual to find direct evidence of blood coagulation in the track produced by the missile in its passage through the lung, and the wall of the track is also infiltrated, to a varying depth, by extravasated blood. Henry and Elliott record in their series four cases of perforating wounds of the lower chest wall involving the pleural cavity below the area occupied by the lung and not wounding the lung, and where no haemothorax existed. Many instances of extensive injury to the chest wall opening the pleura have been seen where the lung itself was not involved, and where no haemothorax was present. The great rarity of secondary haemorrhage in cases of haemothorax is also in favour of the pulmonary origin of the bleeding, since the collapse of the lung associated with the haemothorax tends not only to check haemorrhage directly but also to prevent secondary haemorrhage owing to the rarity of septic inflammations in collapsed lung. It is probable, therefore, that in the great majority of cases of haemothorax the source of the bleeding is the injury to the lung, although cases may be seen occasionally where the haemorrhage is derived from a vessel in the chest wall, such as an intercostal or the internal mammary artery, or one of the big vessels at the root of the neck. Injuries of the great vessels of the neck sometimes coexist with haemothorax; aneurysm of the innominate artery has been seen in association with pneumo-haemothorax, but in such cases it is probable that the haemothorax is really independent of the arterial lesion and due to other pul. monary lesions, and the haemothorax or pneumo-haemothorax may be on the opposite side to that of the arterial lesion.

In rare instances the haemothorax fluid contains, in addition to blood, bile. The bile is derived from the wound track involving the liver, and in such cases the bile may reaccumulate in the pleural cavity after paracentesis has been performed and the haemothorax fluid drawn off. Repeated paracentesis may be required to draw off the reaccumulations of bilious fluid, and it is remarkable that jaundice does not necessarily develop in such cases, although the amount of bile present in the pleural cavity may be very large. This is further evidence that the pleural cavity in cases of haemothorax does not readily absorb fluid, and the lack of absorptive power is probably in part dependent upon the fibrinous coating on its surface, and in part, perhaps, on the lack of efficient respiratory movement on the injured side. In one case there was evidence of the presence of stomach contents in the haemothorax fluid.

Stgns and Symptoms of Haenothorax.

Dyspnoea, although often at first urgent, diminishes rapidly, and even when the quantity of fluid in the chest is large-that is, over three pints-it is not usually a marked feature after the first three days. It is, however, greatly increased by exertion and movement, and hence many patients on arrival at a base hospital after a long journey often have considerable dyspnoea and distress for twentyfour hours. Moderate pyrexia is usually present, and it may rise to $103^{\circ} \mathrm{F}$; ; but the pulse is not much accelerated, and is usually under 100 , and the patient does not have much distress unless cough is severe, frequent, and painful. The continued presence of high fever, pain, distress, rapid pulse, and furred tongue should always suggest the possibility of the haemothorax being infected, and a sample of the fluid should be removed with an exploring syringe without delay in order to determine this point by bacterio. logical methods. Jaundice is an important sign, as, if marked, it is a characteristic sign of infection, and more especially infection with anaërobic bacilli. In simple sterile haemothorax only a slight icteric tinge is present in the conjunctiva, but in anaërobic infections a deep yellow jaundice involving the skin generally develops, often with great rapidity. Such a jaundice is often mis. interpreted as indicating a wound of the liver, especially when the wound is in the lower chest, but jaundice is quite exceptional in liver wounds, whereas it is a very characteristic sign of infected wounds, and especially of anaërobic infections. It may also, of course, be seen in the course of streptococcal infections.

The physical signs produced by haemothorax present considerable variety and often are very difficult to interpret. They are much more complex than those usually regarded as characteristic of simple pleural effusion.

One outstanding sign, easily demonstrated by percussion and confirmed by $x$-ray examination, is that the diaphragm on the affected side is much higher than normal. The high level of the diaphragm is most easily demonstrated in left-sided haemothorax, since then the tympanitic percussion note due to the stomach resonance is readily obtained high up in the axilla, and also in the antero-lateral region of the chest. In some instances this tympanitic stomach resonance may reach so high a level as to merge into the area of skodaic resonance in the infraclavicular region above the level of the fluid, and unless care is taken a mistaken diagnosis of pneumothorax may readily be made. $X$-ray examination often reveals that the diaphragm is not only high but also immobile. The skodaic resonance obtained in the upper chest above the level of the fluid is usually much more marked and much more extensive in its distribution than that present in ordinary pleural effusion, and not infrequently extends beyond the middle line, and this fact again is liable to lead to confusion of the condition with pneumothorax. The distribution of the skodaic resonance is confirmed by the observations on the morbid anatomy, as these show that the upper portions of the lung, especially anteriorly, are greatly over-distended and emphysematous, and that quite frequently the upper lobe is so over-distended as to extend well beyond the mid-sternal line.

The high level of the diaphragm, notwithstanding the presence of $a$ large amount of fluid in the chest, and the very extensive and marked skodaic phenomena are two stiviking features of haemothorax and are in contrast to what obtains in simple pleural effusion. Over the area occupied by the fluid, blowing tubular, or even cavernous, breath sounds are frequently heard on auscultation. ' They are not only more marked than those occasionally heard over pleural effusions, but they are also heard over a much larger area, instead of being limited, as in pleural effusion, to a small area in the vicinity of the angle of the scapula.

Bronchophony and oegophony are also well marked and very definite; pectoriloquy is by no means rare. The physical signs thus often present a more or less clear resemblance to those usually regarded as characteristic of pneumonic consolidation, and it is not surprising that in a considerable number of cases where haemothorax is present, the erroneous diagnosis of so-called traumatic pneumonia is made. The signs described above are not present in all cases; in some the signs are similar to those found in cases of pleural effusion-that is, dullness, weakness, or absence of vocal fremitus, and weal or absence of breath sounds. Further, a case may present at an early period of its course the first set of signs with well-marked tubular breathing, and later on, with an increase in the amount of fluid, the second set with weak or absent breath sounds. This is more especially observed in infected cases where a rapid and considerable increase of the effusion may occur. In others where, as a result of the presence of anaërobes, gas is formed, a cracked-pot percussion note may be elicited often over a localized area wliere previously the percussion note had been dull. In these cases a bell sound may or may not be obtained, but the percusision note is quite characteristic. 
Collapse of the Lung.

In ordinary pleural effusion the chest on the affected side is usually obviously enlarged, and the respiratory movements are diminished in amplitude; this is also true of some cases of haemothorax, more especially when the amount of fluid present is very large - that is, four to five pints. In a considerable number of cases, however, a very different condition is present, inasmuch as the chest is flattened and retracted on the side of the effusion, and the movements are so much diminished that the affected side is practically immobile. This retraction and immobility of the side may be present not only when the amount of fluid is small, but also in many cases where there is a moderate collection of fluid, approximately two pints in amount, but it does not occur in association with the largest collections. It is remarliable that in cases where retraction and flattening are quite marked, the apex beat may be displaced, as in ordinary pleural effision, towards the opposite and un. affected side. This retraction of the injured side is a remarlable and common phenomenon in cases of haemothorax, and it seems to be a fact of the same order as the high level of the diaphragm already noted. Sometimes it is only seen in the first few days after the wound, and then, with an increase in the amount of the effusion in infected cases, it is replaced by bulging of the usual type. Well marked tubular or even cavernous breathing is heard on auscultation in those cases where marked retraction is present, together with bronchophony and pectoriloguy. The retraction of the injured side, the immobility of the chest and the high level of the diaphragm, would all seem to be due to extensive collapse of the lung or of the lower lobe of the lung, a collapse that is out of proportion to the amount of fluid present in the pleura, and is really very probably a collapse that is more or less independent of the presence of the fluid, and has some other mode of origin. It may well be that this collapse is a constant pheno. menon in cases of gunshot injury of the chest, but that when a large haemothorax coexists its presence is naturally attributed to the mere effusion and its real nature is only obvious where it is clearly ont of proportion to the effusion. 'Thus cases of massive collapse of the lung on the side of the injury have been observed by Bradford and Elliott where the amount of the effusion was so small as not to need aspiration, and where the signs cleared up and the lung re-expanded after a short interval. There are, however, other facts that may be adduced in support of the view that the collapse is more or less independent of the effusion. 'Thus it may occur on the opposite side of the chest to that iujured, and not only is this the case, but it is probably of quite frequent occurrence, only the physical signs produced are erroneously attributed to the prescnce of contralateral pneumonia when contralateral collapse is really the condition present. The main distinguishing feature botween the physical signs of pneumonia and those of massive collapse is the pcsition of the heart's apex beat; in pneumonia there is no appreciable displacement, whereas in massive collapse the apex beat is displaced towards the collapsed lung. In an ordinary haemothorax affecting one side it may be impossible to determine clinically whicther pliysical signs on thę opposite side are due to pneumonia or collapse, since if the apex beat is found displaced, the displacement will necessarily be attributed to the presence of the fluid on the injured side. In such cases the diagnosis of contralateral collapse is largely an inference based on the absence of the clinical picture of pneumonia and on the rapidity. with which the signs clear up, but in any given case there may be much doubt unless the diagnosis is confirmed by autopsy.

Contralateral collapse, however, has been observed in cases of wounds implicating the chest wall only, where physical examinations confirmol by $x$.ray observations showed that there was no lesion of the pleural cavity on the wounded side, the injury being a contour wound. Nevertheless, in such cases very extensive massive collapse has been present on the side opposite to that wounded and where there has been no retained missile or other foreign body. In some instances the massive collapse has involved the whole of the left lung and the displacement of thie apex beat into the left axilla has been very marked. Such patients do not present the clinical picture of pneumonia, although the physical sigus in the affected lung are very similar, and the patients' general condition of comparative well-being, together with the absence of high fever, rusty sputum, etc., shows at once that they are not suffering from pneumonia. Further, contralateral collapse has been observed both during operation on the chest and also on autopsy. In one instance an exploratory operation was performed on a case of contralateral collapse under the impression that the physical signs indicated the presence of an intrapulmonary abscess.

Although the mechanism producing collapse of the lung' on the side of the injury and on the opposite side is obscure and cannot be discussed here fully, the presence of this collapse is probably the explanation of the curious and characteristic physical signs so often present in haemothorax, such as the high level of the diaphragm and the frequent presence of tubular breathing, bronchophony, and pectoriloquy over the area of the pleara where fluid is present. This collapse of the lung must be regarded, therefore, as one of the leading, if not the leading, phenomenon of gunshot injuries of the chest. There is some evidence to suggest that it occurs early, soon after the infliction of the wound, as when cases are seen within a few hours of being wounded few physical signs beyond. immobility and deficient air entry on the side of the injury can be detected. Such cases seen again twenty-four hours later may then show the ordinary signs of a haemothorax, but in their earlier phase the signs are often quite in. significant in comparison with the urgency of symptoms, such as distress and dyspnoea.

Displacement of the heart's apex beat is a valuable sign in haemothorax; sometimes it is of a simple character and similar to that seen in pleural effasion-that is, dis. placed to a varying degree away from the side of the haemothorax. In others the displacement is mainly dependent upon the presence of contralateral collapse, and, as mentioned above, in some of these cases, there is no pleural lesion-that is, no fluid, air, or gas in the plcura on the side of the injury - such cases may be mis. interpreted unless care be taken in the examination. The displacement present in any given case may undergo an increase or a diminution. An increase in displacement is usually due to the haemothorax being really infected, and an increase in the exudation or gas formation has taken place in the interval between the two clinical observations.

A decrease in the degree of cardiac displacement in recent cases of haemothorax and occurring during the first week after the date of wounding is also not infrequent, and is often more difficult of explanation. It is commonly attributed to absorption of some of the bloody exudate; this, however, is highly improbable, since absorption from the ploura in these cases is extraordinarily slow and certainly does not occur to any appreciable extent in the early days after the wound. 'The return of the heart may be due to an alteration in the distribution of the haemo: thorax fluid dependent on increasing collapse of the lung on the side of the lesion, or else it may be due to disap-. pearanee of contralateral collapse. The degree of dis. placement of the heart in any given case is therefore a very uncertain guide of the size of a haemothorax.

\section{Diagnosis.}

The main problem in diagnosis is the determination whether a haemothorax is infected or not, and this often presents difficulties, since a baemothorax that at first runs an apparently sterile course may be infected later, owing to the spread of infection either from the wound track or from some localized infection round a retained foreign body. In some of these cases of delayed infection, and more especially in those with anaërobic infection, the symptoms may develop with much rapidity, and be of such a character as to suggest the occurrence of secondary haemorrhage, where no haemorrhage has taken place. Rapid pulse, pallor, sweating, and collapse are not uncommon symptoms of a rapidily spreading anaërobic infection. Microscopic and bacteriological examination of the fluid withdrawn from the chest will generally determine absolutely the presence or absence of infection, provided care be taken to repeat the exploration and too much stress is not laid on the negative result obtained at the first puncture. It is often necessary to explore the chest at different levels. Cases are seen occasionally where the symptoms, and especially the character and duration of the pyrexia, suggest that infection is present, yet no bacteriological confirmation is obtained eren with 
repeated paracentesis. Such cases may get well with no special treatment after running a prolonged course of several weeks' duration. Their explanation is difficult, but it may be that the pyrexia in such cases is dependent upon the inflammatory process in the wound track, and more especially in that in the lung, and that the layer of fibrin coating the pleural surfaces prevents the infection reaching the pleural fluid. Care must always be taken in considering the diagnostic significance of pyrexia that it is not due to some associated complication, such as purulent bronchitis, contralateral pleurisy, pneumonia, or pericarditis, although all such complications are much more liable to occur in infected than in sterile haemothorax. The rarity of pulmonary inflammation in the compressed lung on the side of the haemothorax should alvays suggest, if symptoms are urgent, that infection of the fluid is present rather than pneumonia, although the physical signs may be such as to render diagnosis difficult. In all such cases there should be no delay in making an exploratory puncture, as it is essential for the successful treatment of infected haemothorax that the cases should bc recognized as early as possible.

Treatuent.

In sterile haemothorax, if the amount of the bloody effusion is small, there is no need for any special active treatment; such cases do well, although their progress may sometimes be slow. If the effusion is at all large in amount-that is, the dullness reaching above the angle of the scapula-the fluid should be removed by aspiration about the end of the first week after the wound. In a few cases earlier aspiration may be required to relieve distress arising mechanically from the amount of fluid present. Aspiration with oxygen replacement is better than simple aspiration, since by this method, with suitable local anaes thetics, the operation can be carried out without discomfort to the patient and without any of the symptoms that so commonly occur in ordinary aspiration, and not infrequently prevent by their urgency the completion of the procedure. Further, with oxygen replacement practically all the fluid present in the chest can be remored at one sitting; this is rarely feasible with ordinary aspiration, where the development of such symptoms as a sense of constriction, distress, cough, etc., occurs beforc all the fluid has been removed. These unpleasant symptoms develop as a consequence of a too sudden change in the intrapleural pressure resulting from the rapid removal of fluid, together with incomplete adjustment owing to incomplete expansion of the lung. Different patients vary considerably in the degree of their tolerance of pleural pressure changes, and these are necessarily much influenced by the degree and rapidity with which the lung re-expands. With an oxygen replacement apparatus a measured quantity of oxygen at any desired pressure can be introduced, and thus violent and sudden clsanges in the intrapleural pressurc are avoided.

For practical purposes it is not necessary to measure accurately the intrapleural pressure; it is sufficient to vegulate the introduction of oxygen by the presence or absence of symptoms. The aspiration of the fluid should be temporarily stopped when distress or a sense of constriction is first noticed by the patient; if the symptoms do nat subside at ouce, a small quantity of oxygen from the oxygen replacement apparatus (Parry Morgan or other) should be allowed to flow into the pleural cavity. Then with the relief of all distress, a further quantity of the fluid can be aspirated and the process continued until the operation is completed. The patient is left at the end of the aspiration with a small quantity of oxygen in the pleura-for example, 200 to 500 c.cm. at a pressure considerably less than the pressure of the haemothorax, but still appreciably above the normal pleural pressure. 'The oxygen is gradually absorbed in the course of the following week, but it is remarkable that many days are required for the pleura to absorb even small quantities of gas, and this slow absorption is probably due to the coating of the parietal and visceral pleura with fibrin, as it is in remarkable contrast to the rapid absorption of air that is knowu tc occur after opening the normal pleura.

Frec drainage is required in all cases of infected haemothorax, and it is of the utmost importance that this should be provided as edirly as possible, as the organization of the deposit of lymph on the visceral pleura produces a great inpediment to the rapid expansion of the ling after the removal of the fluid, and this organization proceeds rapidly in cases where delay in operating takes place. Removal of the infected fluid by free drainage is, however, not sufficient treatment in all cases; the inflammation in the pleural sac and the septic clots must be treated by the local application of some efficient antiseptic, applied either by periodical washing out of the cavity or else by the instillation method and the Carrel-Dakin technique that is now so much used. If the cavity is treated by washing out, care must be taken to avoid raising the intrapleural pressure. Septic clots, often of considerable size, can be removed with success by this method. Recently the Carrel-Dakin technique has been applied with success to cases of infected haemothorax, and a few cases have already been so successfully treated that the operation wound required for the excision of the rib has been closed by secondary suture before the evacuation of the patient to England.

Two difficulties are met with in practice in cases of huemothorax. In one group of cases, although the pyrexia and the patient's general condition suggests the presence of infection, bacteriological examination does not confirm this. In another series of cases organisms are detected in the fluid, but there is little or no pyrexia, and the patient does not seem to be very ill. It is probable that some of the cases falling into this second group recover after simple aspiration, but their convalescence is often very slow, and certainly in some instances sucli cases develop serious and even urgent symptoms. For these reasons it is advisable to excise a portion of rib and procure free drainage in all cases of haemothorax where the bacteriological examination reveals the presence of patho: genic organisms, even if the clinical condition of the patient is not such as to suggest infection. As regneds the first group, the position is more difficult, but it is probably wiser to delay opening the pleura until proof of infection in the fluid is obtained, as the pyrexia and even the other symptoms may have some other origin; it is a serious matter to open a sterile haemothorax in view of the possibility of secondary infection.

Care must be taken in selecting the site for excision of a portion of rib to provide really efficient drainage, and the fact that the diaphragm is abnormally high in these cases must be borne in mind, as otherwise the opening will be made too low down.

In some instances the wound of entry or exit com. municates more or less directly with the pleural cavity and duid in variable quantity drains from it; this drainage is rarely satisfactory or sufficient, and such cases require a counter opening in a suitable situation to really drain the effusion.

1 Bradford and Elliott: British Journal of Surgery, vol. iii, No. 10 1915. 2 Journal of the R.A.M.C., vol. xxvii, No. 5, p. 525.

\section{FACTORS IN FIELD AMBULANCE WORK WHICH HELP THE WORK IN CASUALTY CLEARING STATIONS.:}

By Captain K. M. WaLKER, R.A.M.C.

Is spite of its necessary limitations, the surgery of the field ambulance is of the greatest importance. The treat. ment that a shattered limb receives during the first four or five hours may alter the whole future history of the case.

The surgery of the aid post and the dressing station is necessarily a surgery of emergency. Collection of the wounded, and rapid evacuation, must always be recognized as the most important functions of the field ambulance uuits; and when it is possible to do so, anything in the way of operative treatment must be postponed until the arrival of the wounded man at the casualty clearing station.

Haemorrhage.

In this surgery of emergency the treatment of haemorrhage takes first place, and there is a certain type of case in this connexion to which I should like to call attention. I refei to those cases in which there is a slow, continuous oozing that is at no time sufficiently rapid to be regarded

* Paper read at a meeting of medical officers of an army in the 